\title{
Predicting the outcome of islet isolation in large mammals. Reply to Hubert T, Gmyr V, Vantyghem MC et al. [letter]
}

\author{
P. Andrades • C. K. Asiedu • J. M. Thomas
}

Received: 27 October 2008 / Accepted: 27 October 2008 / Published online: 19 November 2008

(C) Springer-Verlag 2008

To the Editor: We are grateful to Hubert et al. [1] for their comment on our recent publication in Diabetologia [2]. Much effort has been made to identify factors or variables that improve pancreatic islet yield and promote successful islet isolation [3, 4]. Adequate in vivo functional islet mass in the pancreas donor is one of many factors that can have an impact on the outcome of pancreatic islet isolation. Therefore, we agree with Hubert et al. [1] that a reliable method to screen functional donor islet mass in vivo has the potential to improve successful islet isolation outcomes. Other tests, such as glucose tolerance tests, beta cell secretory capacity [5], positron-emission tomography [6], and glucose- or arginineinduced acute insulin release (AIR), have been used to assess functional islet mass. Of these, arginine-induced AIR (AIRarg) is a logical choice for this purpose because it is simple and non-invasive. AIR has been used to measure functional beta cell mass in normal individuals as well as in pancreas/ islet transplant recipients [7].

The preliminary data presented by Hubert et al. demonstrating that AIRarg permitted prediction of successful islet isolation are encouraging [8, 9]. However, we caution that further testing in humans is necessary because the reported data were derived from a relatively limited

P. Andrades $(\bowtie) \cdot$ C. K. Asiedu $\cdot$ J. M. Thomas

VH L107, 1670 University Boulevard,

Birmingham, AL 35294-0019, USA

e-mail: patoandrades@gmail.com number of subjects $(n=29)$. As far as we know, there is no published standardised procedure for the AIRarg test that correlates AIRarg with functional beta cell mass for nonhuman primates (NHP). Clearly it will be useful to establish a standard AIRarg test for NHP to evaluate whether the test can be used to predict successful islet isolation in these species. Since a standardised test will allow results from different laboratories to be compared, the AIRarg test has the potential to predict successful islet isolation and functional transplantable islet mass.

Pancreatic islet transplantation remains a motivating challenge for scientists and a hopeful dream for diabetic patients. Rather than a useless sacrifice of NHP, these valuable animals have given us the wonderful opportunity to understand further the complexity of pancreatic islet isolation and transplantation. A single variable might have a significant impact on the final result, but when weighed in conjunction with all the other variables involved, its effect may change dramatically. This is clearly demonstrated in the univariate and multivariate analysis performed in our study [2]. We will have to wait for further improvements in donor selection, islet isolation and implantation in order to have a reliable procedure to offer to our patients.

Duality of interest J. M. Thomas is a consultant for Genzyme Corporation. The remaining authors declare that there is no duality of interest associated with this manuscript. 


\section{References}

1. Hubert T, Gmyr V, Vantyghem MC, Kerr-Conte J, Pattou F (2008) Predicting the outcome of islet isolation in large mammals doi:10.1007/s00125-008-1198-2

2. Andrades P, Asiedu CK, Gansuvd B et al (2008) Pancreatic islet isolation variables in non-human primates (rhesus macaques). Diabetologia 51:1236-1244

3. Zeng Y, Torre MA, Karrison T, Thistlethwaite JR (1994) The correlation between donor characteristics and the success of human islet isolation. Transplantation 57:954-958

4. Hanley SC, Paraskevas S, Rosenberg L (2008) Donor and isolation variables predicting human islet isolation success. Transplantation 85:950-955
5. Rickels MR, Schutta MH, Markmann JF, Barker CF, Naji A, Teff $\mathrm{KL}$ (2005) $\beta$-Cell function following human islet transplantation for type 1 diabetes. Diabetes 54:100-106

6. Eich T, Eriksson O, Lundgren T (2007) Visualization of early engraftment in clinical islet transplantation by positron-emission tomography. N Engl J Med 356:2754-2755

7. Robertson RP (2004) AIRarg and AIRgluc as predictors of insulin secretory reserve. Transplant Proc 36:1040-1041

8. Hubert T, Jany T, Marcelli-Tourvieille S et al (2005) Acute insulin response of donors is correlated with pancreatic islet isolation outcome in the pig. Diabetologia 48:2069-2073

9. Hubert T, Strecker G, Gmyr V et al (2008) Acute insulin response to arginine in deceased donors predicts the outcome of human islet isolation. Am J Transplant 8:872-876 